\title{
Treatment of tuberculous meningitis in adults: Is the duration of intensive-phase therapy adequate?
}

\author{
S Moodley, ${ }^{1} \mathrm{MB}$ ChB; M S Dlwati, ${ }^{1,2} \mathrm{MB}$ ChB, FC Neurol (SA); A I Bhigjee, ${ }^{1,2} \mathrm{PhD}, \mathrm{MRCP}$ \\ ${ }^{1}$ Department of Neurology, Inkosi Albert Luthuli Central Hospital, Durban, South Africa \\ ${ }^{2}$ Department of Neurology, Nelson R Mandela School of Medicine, College of Health Sciences, University of KwaZulu-Natal, South Africa
}

Corresponding author: S Moodley (sharmoodley@gmail.com)

Tuberculous meningitis (TBM) results in considerable morbidity and mortality, especially in developing countries such as South Africa. Treatment regimens have been extrapolated from treatment for pulmonary tuberculosis, and the intensive-phase duration of 2 months may be inadequate for treatment of patients with TBM. We highlight this situation with a case report of a patient with TBM whose illness progressed after institution of the maintenance phase of treatment. We propose that the intensive-phase treatment of TBM be revisited with regard to duration of treatment, choice of drugs during continuation-phase therapy, or both.

S Afr Med J 2021;111(5):412-415. https://doi.org/10.7196/SAMJ.2021.v111i5.15422

Tuberculous meningitis (TBM) is the most frequent and devastating form of central nervous system tuberculosis, resulting in considerable morbidity and mortality despite advances in antituberculosis agents. ${ }^{[1,2]}$ The outcome depends on the stage of disease when treatment is commenced, with mortality of $15-50 \%$ in HIVnegative patients and $25-80 \%$ in those who are HIV-infected. ${ }^{[3]}$

Other reasons for the poor prognosis include delayed diagnosis due to the nonspecific early symptoms, poor sensitivities of diagnostic tests, challenges regarding the current therapeutic regimens, and the host response to the infection. These problems have been highlighted in recent publications. ${ }^{[4,5]}$

There is little evidence to guide optimal treatment in TBM, ${ }^{[6]}$ with the current choice of drugs, their dosages and the duration of treatment based on pulmonary tuberculosis (PTB) treatment.

Current South African (SA) and World Health Organization tuberculosis guidelines recommend 9 months' treatment for TBM, consisting of intensive-phase therapy for 2 months with a combination of rifampicin, isoniazid, ethambutol and pyrazinamide, followed by 7 months of rifampicin and isoniazid as first-line treatment. ${ }^{[7]}$ Apart from a few studies ${ }^{[8,9]}$ using 3 months, the duration of the intensive phase of treatment is 2 months. The total duration of antituberculosis therapy (ATT) in TBM varies from 9 to 18 months.

We have not infrequently seen patients improve during the intensive phase of treatment only to relapse once switched over to the continuation phase (unpublished observations). A patient recently admitted to our unit highlights this issue.

\section{Case report}

A 25-year-old HIV-negative woman presented to a regional hospital with a 1-month history of headaches, nausea, photophobia, phonophobia and associated night sweats. She was confused, with meningism and no focal neurological deficits. The disease severity at this point was classified as modified Medical Research Council (MRC) grade $1 .^{[3]}$

The initial cerebrospinal fluid (CSF) examination revealed CSF glucose $1.6 \mathrm{mmol} / \mathrm{L}$ and protein $2.31 \mathrm{~g} / \mathrm{L}$, with 27 cells $/ \mu \mathrm{L}$ (3 polymorphonuclear cells and 24 lymphocytes), Xpert MTB/RIF Ultra
(Cepheid, USA) was positive with positive sensitivity to rifampicin The cryptococcal antigen was negative. The patient was commenced on intensive-phase ATT without steroids. She reported symptomatic improvement, and at 8 weeks she was changed to continuation-phase ATT (rifampicin and isoniazid).

One week later, while she was on continuation-phase therapy, she presented with worsening severe headaches with vomiting. She also reported painful feet, with weakness in the lower limbs and lower back pain. Vision, swallowing and hearing were normal and the sphincters were reported as intact. She was transferred to the neurology unit at Inkosi Albert Luthuli Central Hospital, Durban, for further management.

On examination she was alert and co-operative with unremarkable findings on general examination. Neurological assessment revealed neck stiffness with a normal mental state. She had bilateral esotropia on primary gaze, with bilateral abduction deficits. The pupils were equal and reactive to direct light, and fundoscopy was normal. She had a flaccid quadriparesis with distal more than proximal weakness and was not ambulant. She had a thoracic level (T3) sensory level to all modalities. Disease severity was now classified as modified MRC grade $2 .{ }^{[3]}$

A chest radiograph was normal. Magnetic resonance imaging (MRI) of the brain (Figs 1, 2 and 3) revealed a focal area of whitematter hyperintensity in the left temporal region and dilated occipital horns of lateral ventricles on a T2-weighted axial study. Post-contrast brain imaging showed features of diffuse leptomeningeal and basal meningeal enhancement with multiple punctate enhancing lesions involving the cerebral hemispheres and cerebellum. MRI of the spine (Fig. 4) showed extensive meningeal enhancement with enhancing lesions in the brainstem.

Repeat CSF examination revealed a protein level of $7.16 \mathrm{~g} / \mathrm{L}$, CSF glucose $4.7 \mathrm{mmol} / \mathrm{L}$ (plasma glucose $5.7 \mathrm{mmol} / \mathrm{L}$ ), and $868 \mathrm{cells} / \mu \mathrm{L}$ (100 polymorphonuclear cells, 608 lymphocytes, 160 erythrocytes). The CSF was yellow in colour and Xpert Ultra was negative. HIV enzyme-linked immunosorbent assay was negative.

The patient was recommenced on intensive-phase ATT. Oral prednisone was added and inpatient rehabilitation was commenced. 


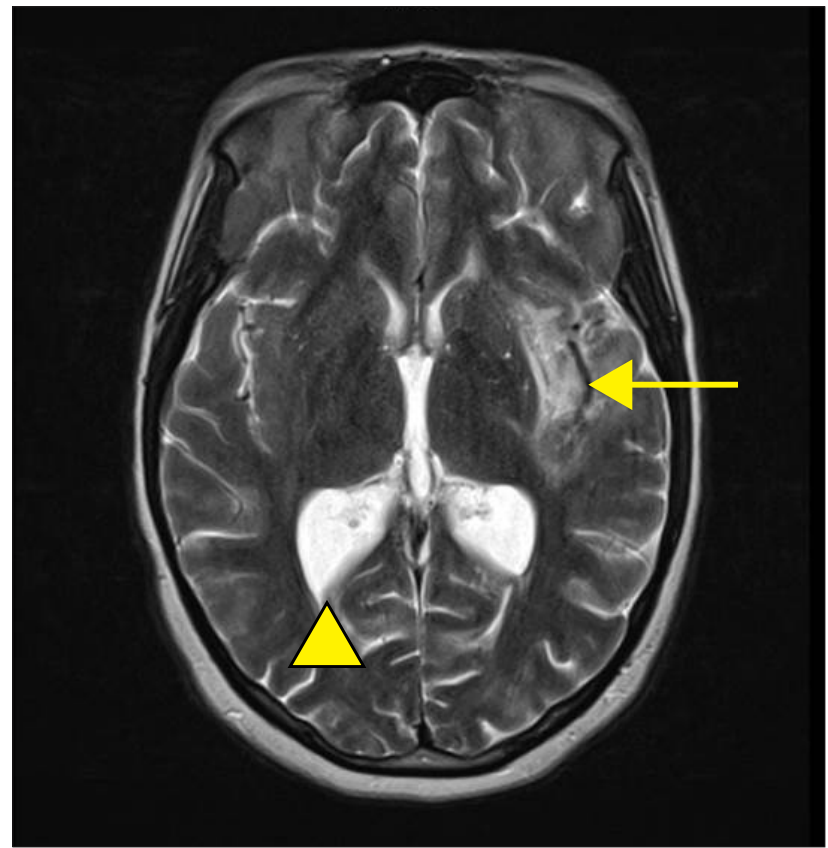

Fig. 1. T2-weighted axial image showing focal area of white-matter hyperintensity in the left temporal region (arrow) and dilated occipital horns of lateral ventricles (arrowhead).

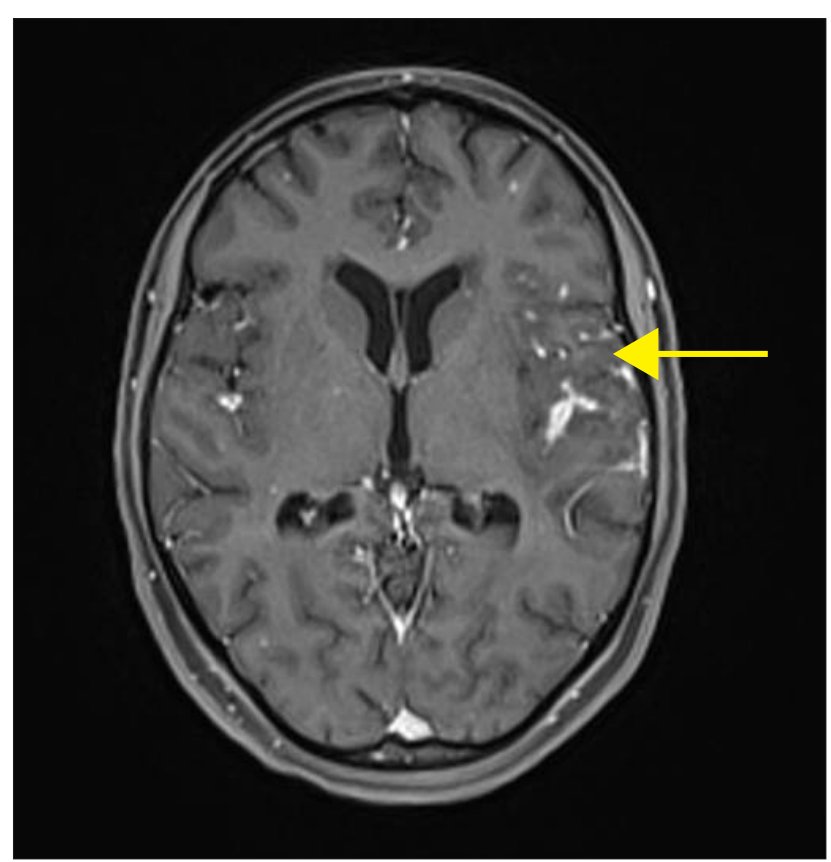

Fig. 2. Features of diffuse leptomeningeal enhancement marked in the left temporal region (arrow).

On review 2 months after re-initiation of intensive therapy (i.e. 4 months from diagnosis), she had improved dramatically and was able to walk.

\section{Discussion}

The Tuberculous Meningitis International Research Consortium discussed knowledge gaps in antimicrobial therapy at a meeting in Lucknow, India, but no mention was made of the duration of the intensive phase of treatment. ${ }^{[4]}$ Similarly, a recent review reported current ongoing therapeutic trials, none of which entailed re-examining the duration of the intensive phase of treatment. ${ }^{[5]}$

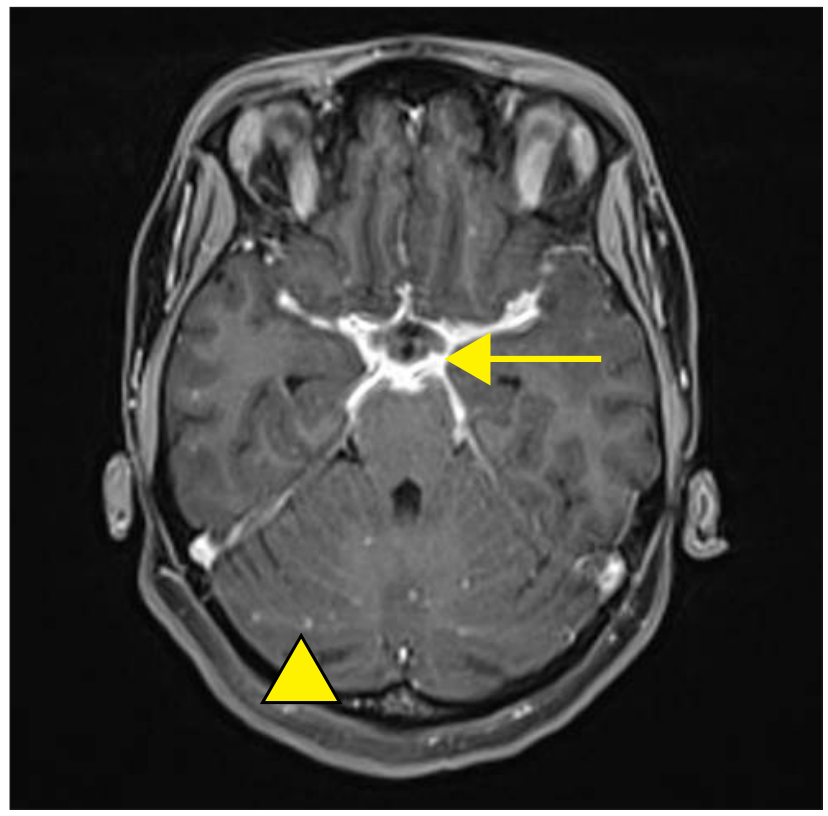

Fig. 3. Basal meningeal enhancement (arrow) with multiple punctate enhancing lesions diffusely involving the cerebral hemispheres and cerebellum (arrowhead).

The three most important elements by which treatment response can be measured are early morbidity, mortality and relapse rates. ${ }^{[10]}$ The ideal ATT must fulfil two key requirements: enhanced activity against Mycobacterium tuberculosis, plus ability to achieve an adequate CSF concentration. ${ }^{[11]}$ In evaluating regimens for the management of PTB, relapse rates of 5\% are often considered satisfactory; however, in the case of TBM it must be ascertained whether any risk of relapse is acceptable. ${ }^{[10]} \mathrm{A}$ study by Pusch et al ${ }^{[12]}$ reviewed the long-term mortality of extrapulmonary tuberculosis syndromes. They found that the therapy duration associated with lowest mortality for TBM was 8 months, and that thereafter mortality increased with longer duration of therapy. This was a small retrospective study and intensive-phase therapy durations were not specified.

Furthermore, the current ATT regimen is based largely on expert opinion and does not take into account the differential ability of antituberculosis drugs to penetrate the blood-brain barrier (BBB) or blood-cerebrospinal fluid barrier, potentially resulting in suboptimal CSF concentration, disease site exposures, bacterial killing and treatment outcomes. ${ }^{[13]}$

Isoniazid and pyrazinamide have good CSF penetration, with CSF concentrations of $80-90 \%$ and $90-100 \%$, respectively, and display treatment-shortening ability. ${ }^{[11]}$ Isoniazid has early bactericidal activity and is metabolised by polymorphic $\mathrm{N}$-acetyltransferase 2 with two phenotypic patterns: fast and slow metaboliser. Slow acetylators have been noted to have high plasma and CSF concentrations. ${ }^{[13]}$ CSF penetration of total rifampicin is poor and penetration of ethambutol is the poorest, with CSF concentrations of $10-20 \%$ and $20-30 \%$, respectively, even with an inflamed BBB. ${ }^{[11]}$

Rifampicin at a dose of $450-750 \mathrm{mg} / \mathrm{d}$ appears to be well tolerated, with $\sim 3 \%$ of adverse reactions reported to require discontinuation, even when combined with isoniazid. The incidence of clinical hepatitis ranges from $2 \%$ to $11 \%$ of patients treated with rifampicin, isoniazid and pyrazinamide, which appears to be idiosyncratic but could be dose related in the presence of pre-existing liver disease. ${ }^{[14,15]}$ Prolonged use of intensive-phase ATT requires caution, especially in patients with risk factors predisposing to possible adverse events. 
Early aggressive ATT results in early improvement and is based on the anticipation that incomplete treatment may lead to increased rates of death, relapse and neurological sequelae. ${ }^{[16]}$ On the other hand, prolonged treatment adds to the adverse effects of medications and can lead to poorer compliance.

The intensive phase of treatment may be a potential point of intervention for improving outcome. A number of studies have studied or are studying different drug regimens for the intensive phase of treatment.

Various clinical trials have investigated hyper-intense ATT with either intravenous rifampicin or a higher dose of oral rifampicin with addition of fluoroquinolones during the intensive-phase therapy. These studies showed no effect on clinical outcome or improvement in survival compared with standard therapy. In contrast, isoniazid exposure was associated with improved
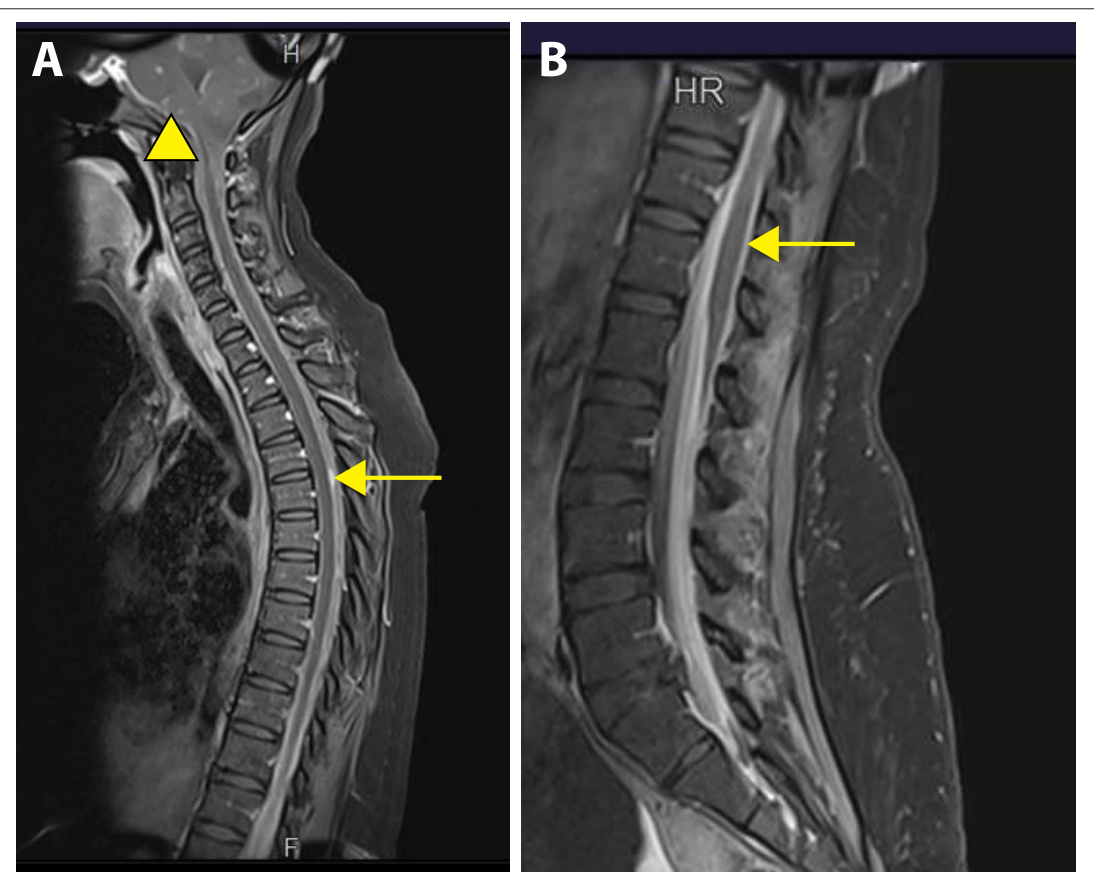

Fig. 4, A and B. Extensive meningeal enhancement (arrows) with enhancing lesions in the brainstem (arrowhead).

survival, while low exposure was predictive of death and was linked to the fastmetaboliser phenotype ${ }^{[13]}$ Higher doses of isoniazid should be investigated, especially in fast metabolisers. ${ }^{[1,10,11,13,17-19]}$ In one randomised controlled trial, standard-dose levofloxacin was compared with standarddose rifampicin, together with isoniazid, pyrazinamide and ethambutol. Patients receiving levofloxacin had improved outcomes, possibly due to better CSF penetration of levofloxacin compared with rifampicin ${ }^{[20]}$

Linezolid is well established for the treatment of PTB and has an additive effect when combined with rifampicin. A retrospective study in adults with TBM suggested a favourable outcome. ${ }^{[21]}$ Agents such as the nitroimidazole, delamanid, may be another reasonable option. ${ }^{[22]}$

Going forward, the options of manipulating the intensive phase of treatment include evaluating drug penetration into the

Table 1. Possible changes in the intensive phase of therapy: Potential regimens
CSF, increasing the duration of intensivephase treatment using the standard agents, and combining the standard drugs with other drugs. Possible variations in the management of the intensive phase are summarised in Table 1.

Our patient was not commenced on corticosteroid therapy at the regional hospital at the time of diagnosis of TBM. Despite this, there was a marked improvement in her symptoms. Deterioration was noted after the change to continuation-phase therapy. Because the clinical deterioration occurred after this change, it was not attributed to a paradoxical reaction. This conclusion is further supported by her continued clinical improvement while on intensive-phase treatment and relapse within a week of introduction of the continuation-phase therapy. Her dramatic clinical improvement when re-initiated on intensive-phase therapy is further evidence against a possible paradoxical reaction.

\section{Conclusions}

Meningitis is the most devastating manifestation of tuberculosis, with high morbidity and mortality. The duration of the intensive phase of treatment of TBM has been extrapolated from data obtained in the management of PTB. The optimal antimicrobial intensive treatment regimen for TBM has not been established in clinical trials. Little attention has been paid to this aspect of treatment.

The patient under discussion highlights the shortcomings of the current treatment guidelines. In our view, the duration of the intensive phase of therapy is too short. Research should be directed towards evaluating a longer duration of intensivephase therapy with the standard drugs or with combinations of different drugs in randomised controlled studies addressing the deficiencies of current regimens.

In the interim, we suggest using intensivephase treatment (rifampicin, isoniazid, pyrazinamide and ethambutol) for a minimum of 3 months.

\footnotetext{
1. Commence RHZE for 3 months, then continue with RH for $6-9$ months $s^{[9]}$

2. RHZE for 2 months, then RHZ as the continuation phase

3. Start with RHZEth for 2 or 3 months, then RH

4. High-dose $\mathrm{R}$ in various formulations and combinations with the other standard agents for 3 months, then standard continuation phase $\mathrm{e}^{[9]}$

5. Levofloxacin with HZE for 2 months, then continue with HZ

6. Commence RHZE with high-dose $\mathrm{H}$ - fast acetylator ${ }^{[13]}$

7. $\mathrm{RHZ}+$ linezolid for $2-3$ months, then continue with RH

8. Delamanid in the future?

$\mathrm{R}=$ rifampicin $; \mathrm{H}=$ isoniazid $\mathrm{Z}=$ pyrazinamide; $\mathrm{E}=$ ethambutol $; \mathrm{Eth}=$ ethionamide $\mathrm{L}=$ levofloxacin.
} 


\section{Declaration. None.}

Acknowledgements. None.

Author contributions. All authors contributed equally.

\section{Funding. None.}

\section{Conflicts of interest. None.}

1. Dian S, Yunivita V, Ganiem AR, Pramaesya T, et al. Double-blind, randomized, placebo-controlled phase II dose-finding study to evaluate high-dose rifampin for tuberculous meningitis. Antimicrob Agents Chemother 2018;62(12):e01014-18. https://doi.org/10.1128/AAC.01014-18

2. Wang MG, Luo L, Zhang Y, Liu X, Liu L, He JQ. Treatment outcomes of tuberculous meningitis in adults: A systematic review and meta-analysis. BMC Pulm Med 2019;19(1):200. https://doi. in adults: A systematic review
org/10.1186/s12890-019-0966-8

3. Wilkinson RJ, Rohlwink U, Misra UK, et al. Tuberculous meningitis. Nat Rev Neurol 2017;13(10):581 Wilkinson RJ, Rohlwink U, Misra UK, et al. Tub
598. https://doi.org/10.1038/nrneurol.2017.120

4. Seddon JA, Wilkinson R, van Crevel R, Figaji A, Thwaites GE; Tuberculous Meningitis International 4. Seddon JA, Wilkinson R, van Crevel R, Figaji A, Thwaites GE; Tuberculous Meningitis International
Research Consortium. Knowledge gaps and research priorities in tuberculous meningitis. Wellcome Open Res 2019;4:188. https://doi.org/10.12688/wellcomeopenres.15573.

5. Donovan J, Thwaites GE, Huynh J. Tuberculous meningitis: Where to from here? Curr Opin Infect Dis 2020;33(3):259-266. https://doi.org/10.1097/qco.0000000000000648

6. Davis A, Meinties G, Wilkinson RJ. Treatment of tuberculous meningitis and its complications in adults. Curr Treat Options Neurol 2018;20(3):5 https://doi.org/10.1007/s11940-018-0490-9

7. World Health Organization. Treatment of Tuberculosis: Guidelines for National Programmes. 4th ed. Geneva: WHO, 2010. https://www.who.int/tb/publications/9789241547833/en/ (accessed 6 April 2021).

8. Brancusi F, Farrar J, Heemskerk D. Tuberculous meningitis in adults: A review of a decade of developments focusing on prognostic factors for outcome. Future Microbiol 2012;7(9):1101-1116. https://doi.org/10.2217/fmb.12.86

9. Donald PR. Chemotherapy for tuberculous meningitis. N Engl J Med 2016;374(2):179-181. https:// 9. Donald PR. Chemotherapy for
doi.org/10.1056/nejme1511990

10. Donald PR. The chemotherapy of tuberculous meningitis in children and adults. Tuberculosis (Edinb) 2010;90(6):375-392. https://doi.org/10.1016/j.tube.2010.07.003

11. Wasserman S, Davis A, Wilkinson RJ, Meintjes G. Key considerations in the pharmacotherapy of tuberculous meningitis. Expert Opin Pharmacother 2019;20(15):1791-1795. https://doi.org/10.1080 /14656566.2019.1638912
12. Pusch T, Pasipanodya JG, Hall RG 2nd, Gumbo T. Therapy duration and long-term outcomes in extra-pulmonary tuberculosis. BMC Infect Dis 2014;14:115. https://doi.org/10.1186/1471-2334extra-p

13. Ding J, Thuy Thuong Thuong N, Pham TV, et al. Pharmacokinetics and pharmacodynamics of intensive antituberculosis treatment of tuberculous meningitis. Clin Pharmacol Ther of intensive antituberculosis treatment of tubercul
2020;107(4):1023-1033. https://doi.org/10.1002/cpt.1783

14. Abulfathi AA, Decloedt EH, Svensson EM, Diacon AH, Donald P, Reuter H. Clinical pharmacokinetics and pharmacodynamics of rifampicin in human tuberculosis. Clin Pharmacokinet 2019;58(9):1103-1129. https://doi.org/10.1007/s40262-019-00764-2

15. Bright-Thomas RJ, Gondker AR, Morris J, Ormerod LP. Drug-related hepatitis in patients treated with standard anti-tuberculosis chemotherapy over a 30-year period. Int J Tuberc Lung Dis 2016;20(12):1621-1624. https://doi.org/10.5588/ijtld.16.0370

16. Vibha D, Prasad K. Prevailing practices in the treatment of tuberculous meningitis (TBM): A cross-sectional study. Postgrad Med J 2019;95(1124):348-349. https://doi.org/10.1136/ postgradmedj-2019-136486

17. Cresswell $\mathrm{FV}$, te Brake $\mathrm{L}$ Atherton $\mathrm{R}$, et al. Intensified antibiotic treatment of tuberculosis meningitis. Expert Rev Clin Pharmacol 2019;12(3):267-288. https://doi.org/10.1080/17512433.2019.1552831

18. Ruslami R, Ganiem AR, Dian S, et al. Intensified regimen containing rifampicin and moxifloxacin for tuberculous meningitis: An open-label, randomised controlled phase 2 trial. Lancet Infect Dis 2013;13(1):27-35 https://doi.org/10.1016/s1473-3099(12)70264-5

19. Yunivita V, Dian S, Ganiem AR, et al. Pharmacokinetics and safety/tolerability of higher oral and intravenous doses of rifampicin in adult tuberculous meningitis patients. Int J Antimicrob Agents 2016;48(4):415-421. https://doi.org/10.1016/j.ijantimicag.2016.06.016

20. Kalita J, Misra UK, Prasad S, Bhoi SK. Safety and efficacy of levofloxacin versus rifampicin in tuberculous meningitis: An open-label randomized controlled trial. J Antimicrob Chemother 2014;69(8):2246-2251. https://doi.org/10.1093/jac/dku103

21. Sun F, Ruan Q, Wang J, et al. Linezolid manifests a rapid and dramatic therapeutic effect for patients with life-threatening tuberculous meningitis. Antimicrob Agents Chemother 2014;58(10):62976301. https://doi.org/10.1128/aac.02784-14

22. Tucker EW, Pieterse L, Zimmerman MD, et al. Delamanid central nervous system pharmacokinetics in tuberculous meningitis in rabbits and humans. Antimicrob Agents Chemother 2019;63:e0091319. https://doi.org/10.1128/aac.00913-19

Accepted 6 January 2021. 